\title{
Análisis del movimiento circular no uniforme mediante el uso de smartphones
} como sensores

Claudio Mario Enrique

\section{RESUMEN}

Este trabajo presenta una propuesta didáctica para analizar el movimento circular de una plataforma usada habitualmente en el estudio de las rotaciones. Se considera que los teléfonos celulares inteligentes pueden ser empleados como herramientas tecnológicas por parte de los estudiantes de ingenierías, favoreciendo el aprendizaje significativo de la Física mediante el uso de las TIC. La experiencia usa como sensor un smartphone con una aplicación $(a p p)$ de acceso libre. Se mide la Fuerza g en las tres dimensiones del espacio ( $\mathrm{x}$; y; z), previa calibración del mismo y selección del sistema de coordenadas cartesianas. Los datos colectados son corregidos, para posteriormente proceder a sus análisis cualitativo y cuantitativo, con el objeto de explicar el comportamiento del sistema plataforma smartphone. Como novedad, se incorpora la relación entre el movimiento circular uniformemente desacelerado y el oscilatorio amortiguado, de manera similar a la existente entre el circular uniforme y el armónico simple.
\end{abstract}

PALAVRAS CLAVE: Movimiento circular; Trabajos Prácticos; Smartphones; Enseñanza de Física. 


\section{INTRODUCCIÓN}

En la enseñanza universitaria de la Física como ciencia experimental, MARTÍNEZ PÉREZ indica que se trata de "desarrollar experiencias de laboratorio con la finalidad de adquirir destrezas y habilidades en el saber conocer y saber hacer" (2015, p. 341).

Por otro lado, la irrupción de las Tecnologías de la Información y Comunicación (TIC) ha generado un gran cambio cultural en todos los órdenes de la sociedad, y el ámbito educativo no debe mantenerse al margen. Por ello deben promoverse acciones y políticas que impulsen su aplicación en el aula de manera didáctica y en función de las necesidades e intereses de alumnos y docentes (ALMIRÓN y PORRO, 2014). Según Aveleyra et al:

[...] la aplicación de las TIC en la enseñanza superior ha comenzado a cuestionar los métodos pedagógico-didácticos empleados hasta ahora, a la vez que han comenzado a aparecer nuevas oportunidades para enseñar y aprender que es necesario aprovechar (2011, p. 2 - 3).

Este paradigma educativo se puede caracterizar por modelos constructivistas de aprendizaje y entornos enriquecidos tecnológicamente (GARCÍA BARNETO y GIL MARTín, 2006). Expresado de otra forma por MAGGIO:

[...] la transformación de las formas de enseñar no se produce por la renovación de los artefactos, sino por la reconstrucción de los encuadres pedagógicos de dicha renovación (2000, p. 110).

Sin embargo, el acceso a las TIC también generaron dificultades (CALDERÓN et al, 2015), principalmente de carácter económico - sobre todo en los países periféricos - debido al costo de los materiales y equipos. En este contexto cobra mucha relevancia el teléfono celular inteligente - más conocido como smartphone - por varias razones. Son usados por los estudiantes como una herramienta de comunicación e información; su acceso masivo permite diseñar actividades educativas de distintas características - entre ellas, como sensores dado que cuentan con acelerómetros, barómetros, giróscopos, etc. Están disponibles muchas aplicaciones (apps) de acceso libre, y sus creaciones y/o modificaciones se dan de manera continua y permanente y se han iniciado numerosas investigaciones sobre su uso en distintas experiencias de Física (KUHN y VOGT, 2013; MARTíNEZ PÉREZ, 2015; SANS et al, 2013; VOGT y KUHN, 2013). Además, y según González et al

[...] estos experimentos realizados por los alumnos con sus propios teléfonos les permiten confrontar sus conocimientos con los resultados de sus medidas, aprendiendo física con la observación de su entorno (2015, p. 1).

Esta propuesta considera fundamental el dominio de aspectos pedagógicos específicos para poder adaptar las propuestas didácticas a su entorno particular de enseñanza y poder realizar nuevos diseños poniendo en juego la creatividad.

Con el uso de los teléfonos inteligentes como herramienta cognitiva, en esta investigación pretendemos ampliar las funciones habituales de dichos dispositivos en los estudiantes de ingeniería para lograr su aprendizaje.

Por otra parte, es habitual - tanto en los textos como en los simuladores de 
movimiento circular uniforme sobre un diámetro" (SEARS et al, p. 423). Pero por otro lado, y de la misma manera, no existen trabajos que relacionen el movimento circular no uniforme y el oscilatorio amortiguado. Esta investigación intenta hacer un aporte en esta dirección.

\section{DESCRIPCIÓN DE LA EXPERIENCIA}

En este trabajo se presenta una actividad experimental, cuya meta es lograr que los estudiantes realicen dichas actividades mediante un enfoque investigativo y articulado tanto con los otros alumnos como con el docente. El smartphone empleado en este trabajo es un Samsung Galaxy Young 2, con sistema operativo Android 4.4.2. Contiene un acelerómetro, el cual es un dispositivo que convierte aceleraciones y cambios de velocidad en una señal eléctrica. Si bien existen de varios tipos, los más utilizados son los capacitivos. Vale citar que en este trabajo no se describirá el principio de funcionamiento del mismo.

La aplicación seleccionada para esta indagación fue Physics Toolbox Suite de Vieyra Software, versión 1.9., disponible en la página web: https://www.vieyrasoftware.net/; junto a información relevante respecto a sus usos. El sensor elegido del menú fue el mide Fuerza g. De manera resumida, dicha fuerza se define como la relación entre las fuerzas Normal y gravitacional (FN / $\mathrm{Fg}$ ) en tres dimensiones. Ésta cambia cada vez que el dispositivo móvil aumenta o disminuye el módulo de la velocidad, o cambia su dirección.

Cuando el smartphone no está acelerado y se encuentra en posición horizontal, lee valores $[0 ; 0 ; 1]$; asociado a las coordenadas $[x ; y ; z]$; respectivamente. Esto significa que una FN sólo se experimenta en la dirección " $z$ ", y que es igual a Fg. Vale aclarar que dicha información se encuentra disponible en la misma app.

Además, debe destacarse que dicha app cuenta con la opción de calibrar el acelerómetro previo a la toma de los datos experimentales.

Para la presentación de éstos, se empleó la visualización gráfica única mediante lectura gráfica para los ejes $x, y, z$; en función del tiempo transcurrido . También se procedió a su captura mediante un archivo de tipo csv (commaseparated values, o valores separados por comas), con una velocidad de recolección Normal $(5 \mathrm{~Hz})$. La sensibilidad de este dispositivo para el tiempo medido es de $1[\mathrm{~ms}]$; mientras que para la fuerza g es $1.10^{-4}$.

Posteriormente, estos valores fueron transferidos a una planilla de cálculo común para proceder a su procesamiento y análisis. Para finalizar, y si bien no se han realizado hasta el presente comparaciones con métodos estandarizados, debe destacarse que esta app presenta resultados confiables en sus mediciones (MARTINEZ PÉREZ, 2015), a tal punto que ha sido empleada como patrón de referencia en otras indagaciones sobre sensores (LENDOIRO VALLE, 2017).

Como sistema que puede girar alrededor de un eje que pasa por su cento de masa, en esta experiencia se hizo uso de una plataforma circular que habitualmente se emplea para el trabajo práctico de Dinámica Rotacional. Consta de un disco superior de radio igual a $35,6 \mathrm{~cm}$, soportado por un cilindro macizo metálico apoyado otro disco cuyo radio es de $51,0 \mathrm{~cm}$, y se apoya sobre el piso 
mediante tres patas regulables. Previo a su uso, la plataforma se niveló para garantizar su horizontalidad. En la misma acción, se calibró el acelerómetro sobre la superfície plana para ajustar los valores iniciales $x, y ; y z$. También se realizaron varias pruebas con el teléfono - a diferentes distancias respecto al centro de la plataforma -. No obstante ello, en este trabajo se presentan los resultados cuando el smartphone se ubicó en el límite del borde exterior del disco inferior.

Antes de realizar las experiencias prácticas en la plataforma se verificaron experimentalmente las direcciones del sistema de coordenadas cartesianas provisto por la bibliografía. Para ello, se movió el teléfono sobre una superficie plana para cada una de las tres direcciones. El movimento de rotación del sistema se hizo en dirección antihoraria, por lo que las direcciones positivas de los ejes de coordenadas cartesianas, cuyo origen se encuentra en el centro de masa (CM) del smartphone, son los mostrados en la Figura 1:

Figura 1: Sistemas de coordenadas cartesianas elegido

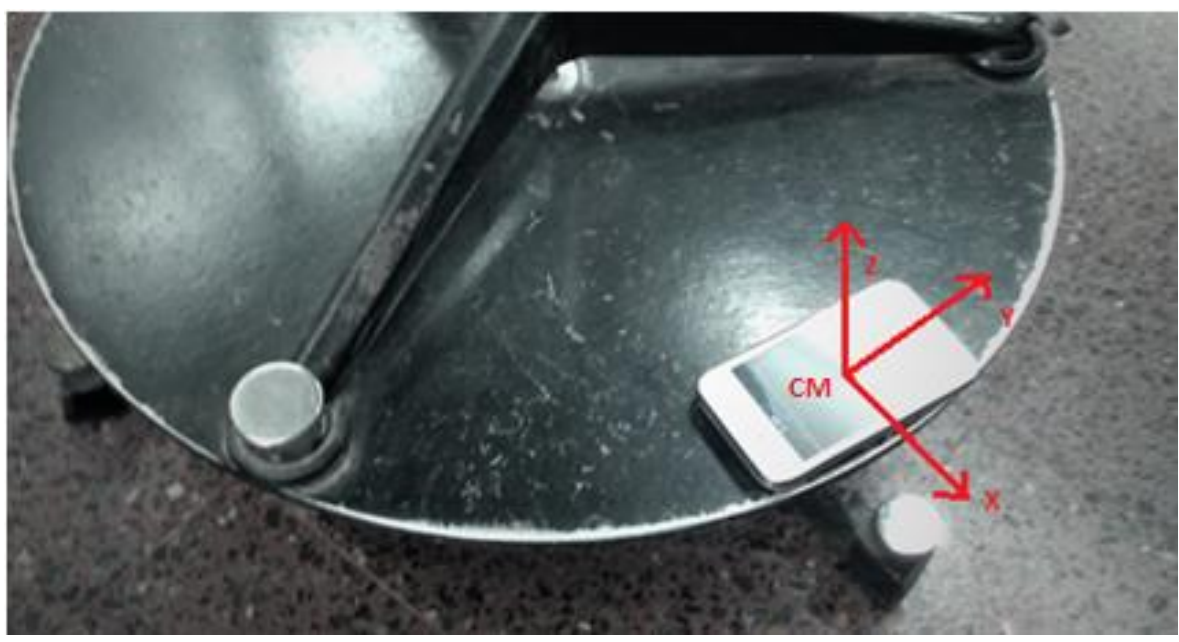

Fuente: autor

Una vez posicionado el sistema, se pulsaron los botones para la recolección de los valores experimentales y se aplico un impulso para sacarlo del estado de equilíbrio, de modo tal que pudo moverse un poco más de 4 vueltas completas y luego se frenó definitivamente. Luego se dio fin a la toma de datos, y se generó un archivo csv que fue importado a una planilla de cálculo.

\section{ANÁLISIS Y DISCUSIÓN DE LOS RESULTADOS}

Los valores hallados de las tres componentes de la Fuerza g (gFx; gFy; gFz), junto a la fuerza neta (TgF) en función del tiempo fueron transferidos desde el archivo .csv correspondiente a la planilla de cálculo, tal como se citó anteriormente. La gráfica obtenida se presenta en la Figura $\mathbf{2}$. 
Figura 2: Fuerzas g vs. tiempo

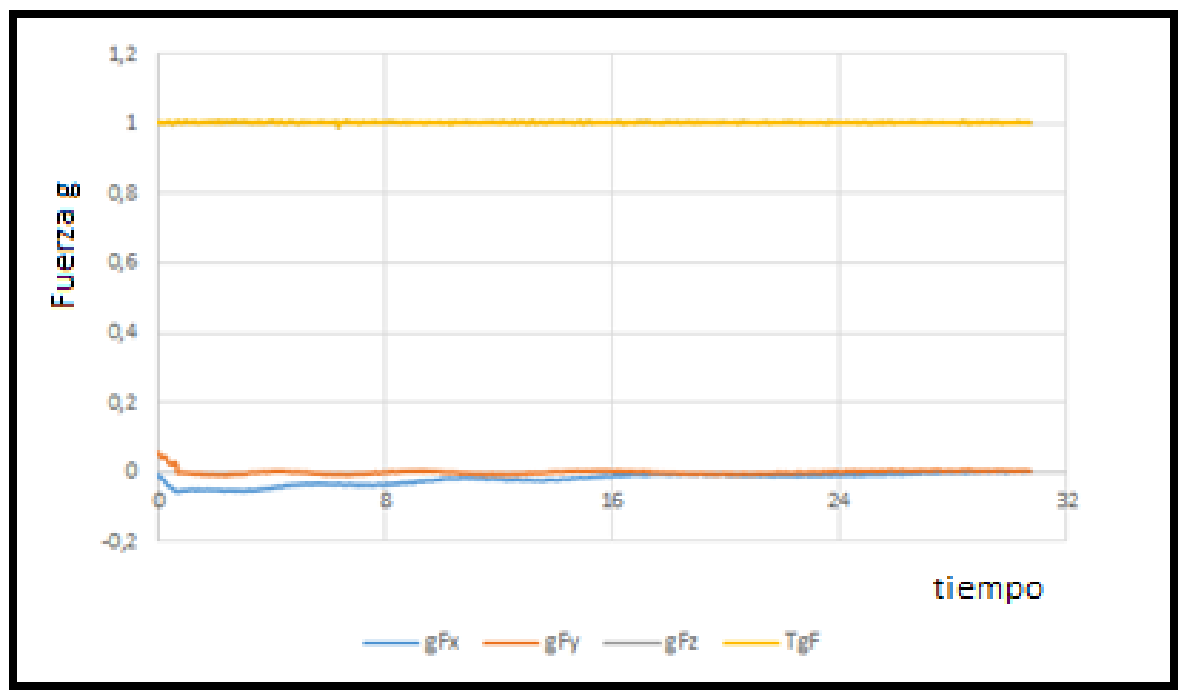

Fuente: autor

Así, se evidencia que estos datos experimentales medidos por el sensor (gFx; gFy) no expresan las componentes de la aceleración de un sistema que describe un movimiento circular no uniforme - centrípeta o radial; y tangencial . Por ello, antes del análisis debe procederse a la corrección de dichos valores. Partiendo de la definición de Fuerza g, haciendo uso de la segunda Ley de Newton, e involucrando un cambio de signo en el eje " $x$ " - dado que la dirección positiva de gFx es negativa para el eje radial del movimiento circular, según la Figura 1 - el cálculo de la aceleración centrípeta (ax) se obtiene de la siguiente manera:

$$
\begin{aligned}
& g F x=\frac{F N}{F g} \\
& F g \cdot g F x=\mathrm{m} \cdot \mathrm{a} \\
& m \cdot g \cdot g F x=m \cdot a \\
& -g \cdot g F x=-a=a x
\end{aligned}
$$

De manera análoga para la dirección tangencial - aunque sin modificar el signo -, se obtiene que dicha aceleración tangencial (ay) es igual a:

$$
a y=g \cdot g F y
$$

Las representaciones gráficas de estos resultados corregidos y adecuados para el análisis del movimento circular no uniforme, se muestran en la Figura 3. Como se trata de un sistema que se ha detenido por el rozamiento en el eje de rotación, el sistema - la plataforma más el smartphone -, describieron un Movimiento Circular Uniformemente Desacelerado. 
Figura 3: Aceleraciones radial (ax) y tangencial (ay) en función del tempo

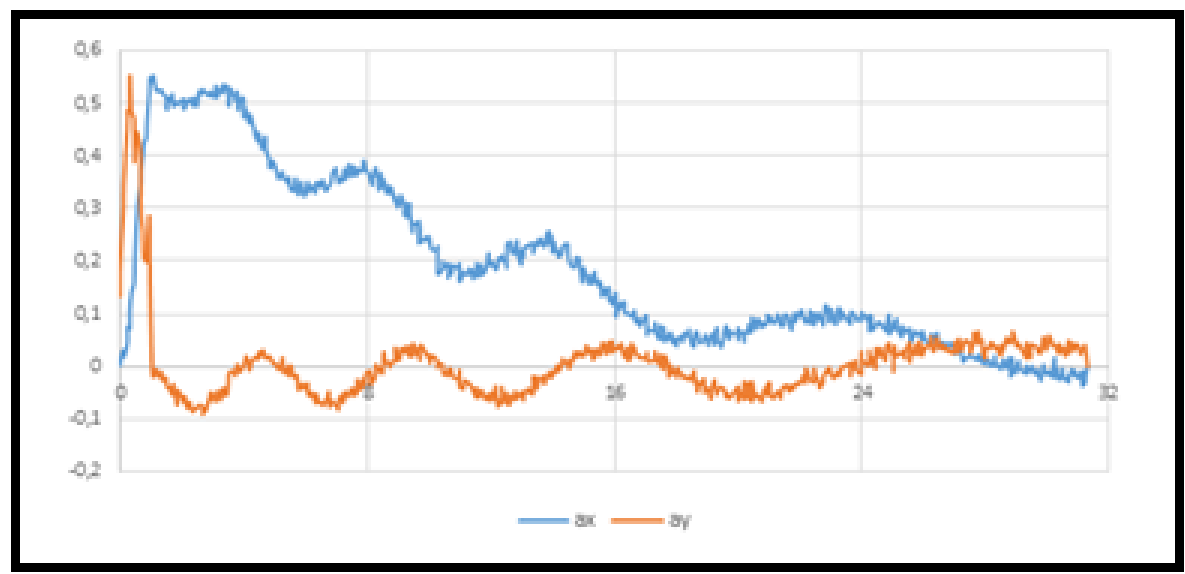

Fuente: autor

Mediante el análisis descriptivo de las representaciones de las aceleraciones en las direcciones tangencial (ay) y radial (ax) según la Figura 3, se observa que:

a. la primera parte de las gráficas revelan un comportamiento rectilíneo desde el origen, punto $(0 ; 0)$, hasta que al llegar a 1,08 $[\mathrm{s}]$, se alcanzan los valores máximos ( $a y=0,552 \mathrm{~m} / \mathrm{s}^{2} ; a x=0,541 \mathrm{~m} / \mathrm{s}^{2}$ ). Es decir, el sistema parte del reposo (la velocidad inicial es nula a tiempo $=0$ ), y debido al impulso aplicado, se produce un incremento lineal de ambas aceleraciones.

b. En la segunda parte, y debido sólo al rozamiento, ambas se detienen definitivamente a los 31,33 [s], pero la representación gráfica indica que lo hacen de manera oscilante y no periódica. Ésta es quizás la información más relevante, dado que las dos aceleraciones resultan ser variables en el tiempo, y es una situación que habitualmente no es desarrollada durante el curso de Física. Por lo tanto, se evidencia aquí una de las ventajas, del uso de los smartphones como sensores, para el movimiento circular no uniforme.

Como continuación en esta investigación, una de las opciones consiste en hallar el módulo de la velocidad tangencial (Vt) en función del tiempo, para un punto del disco inferior de la plataforma a una distancia " $r$ ". Para ello, se emplean los datos de la aceleración radial (ax) presentes en la Figura 3. En particular, la distancia $r$ es la existente desde el centro de masas de la plataforma al centro de masas del smartphone. Finalmente, mediante la ecuación (6) se puede obtener la serie de datos corregidos en la planilla de cálculo, cuya gráfica se presenta en la Figura 4.

$$
V t=\sqrt{a x \cdot r}
$$


Figura 4: módulo de la velocidad tangencial en función del tiempo

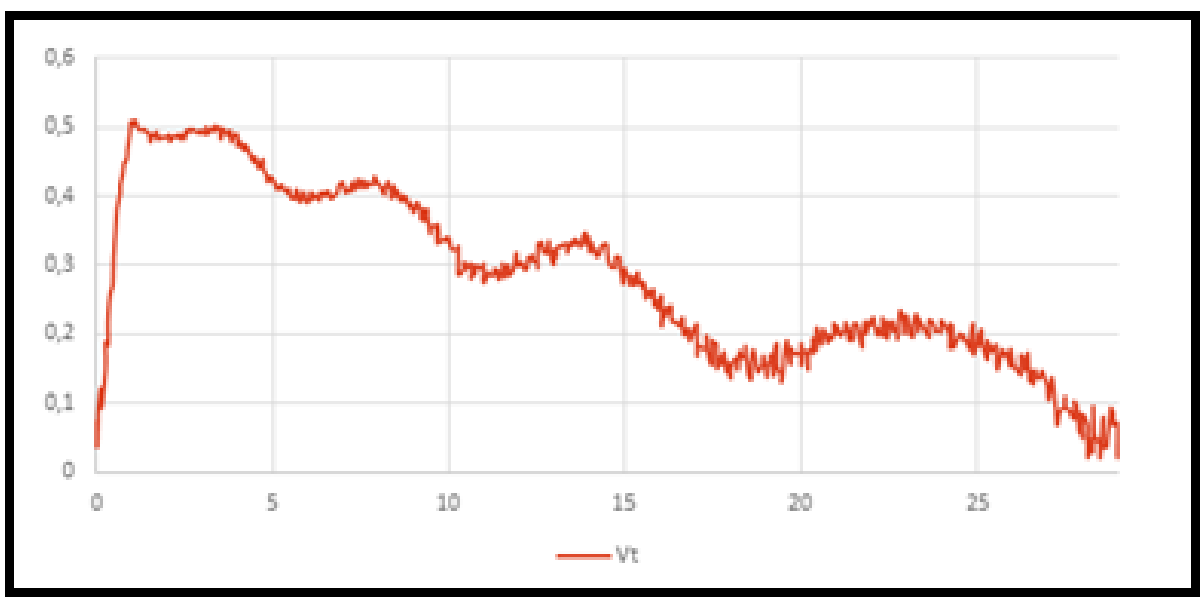

Fuente: autor

Dado que existe una proporcionalidad entre los valores representados en la Figura 4 respecto a la Figura 3, la primera parte muestra que la velocidad tangencial tiene un comportamiento lineal. Después de alcanzar su valor máximo, se observa que el sistema adquiere un comportamiento oscilante y no periódico. Dicho de otro modo, la $v_{t}$ - al igual que la aceleración centrípeta, según la ecuación (6) - disminuye de forma amortiguada cuando se trata de un movimiento circular no uniforme.

\section{RELACIÓN ENTRE EL MOVIMIENTO CIRCULAR NO UNIFORME Y EL OSCILATORIO AMORTIGUADO}

El análisis de las Figuras 3 y $\mathbf{4}$ evidencian otra virtud de esta experiencia de laboratorio, la que consiste en que se puede establecer una relación entre el movimiento circular uniformemente desacelerado y el movimento oscilatorio amortiguado. Esta es una situación que raramente se presenta en los tradicionales libros de Física universitária. Por el contrario, sí se suele explicar la vinculación entre los movimentos circular uniforme y armónico simple, junto a la descripción de la periodicidad de la fuerza - o de la aceleración - centrípeta en función del tiempo. También existen muchos simuladores de libre acceso que pueden representar dicha situación dinámica.

Desde el punto de vista didáctico, para un estudiante de primer año de universidad el estudio del movimiento oscilatorio amortiguado resulta ser complejo, dado que su tratamiento matemático requiere el uso de ecuaciones diferenciales de segundo orden. Por otro lado, es frecuente que los estudiantes no comprendan acerca de su carácter no periódico (ENRIQUE, YANITELLI Y GIORGI, 2018). Con este propuesta educativa, se cree que los sujetos pueden notar las características de un sistema oscilante amortiguado estableciendo una relación con el movimento circular uniformemente desacelerado; análogo a la existente entre el circular uniforme y el oscilatorio armónico simple.

Haciendo uso de una de las varias opciones que se pueden diseñar con esta propuesta, una consiste en analizar la velocidad instantánea de un punto ubicado en el CM del smartphone, en función del tiempo - o a una distancia $r$ del eje de rotación que puede ser fácilmente medida. Para dicho análisis, se deben seleccionar los valores instantáneos de la velocidad tangencial (Vt) de dicho 
punto en función del tiempo $(\mathrm{t})$, provenientes de la planilla de cálculo que permitieron construir la Figura 4. Éstos se presentan en la Tabla 1 y en la Figura 5:

Tabla 1

\begin{tabular}{c|c|c}
\hline $\mathrm{t}(\mathrm{s})$ & $\mathrm{Vt}(\mathrm{m} / \mathrm{s})$ & $\Delta \mathrm{t}$ \\
\hline 1,08 & 0,51 & - \\
5,68 & 0,41 & 4,60 \\
10,98 & 0,30 & 5,30 \\
18,20 & 0,16 & 7,22 \\
28,80 & 0,09 & 10,60 \\
\hline
\end{tabular}

Fuente: autor

Figura 5: velocidades instantáneas (Vt) en función del tiempo con curva de ajuste

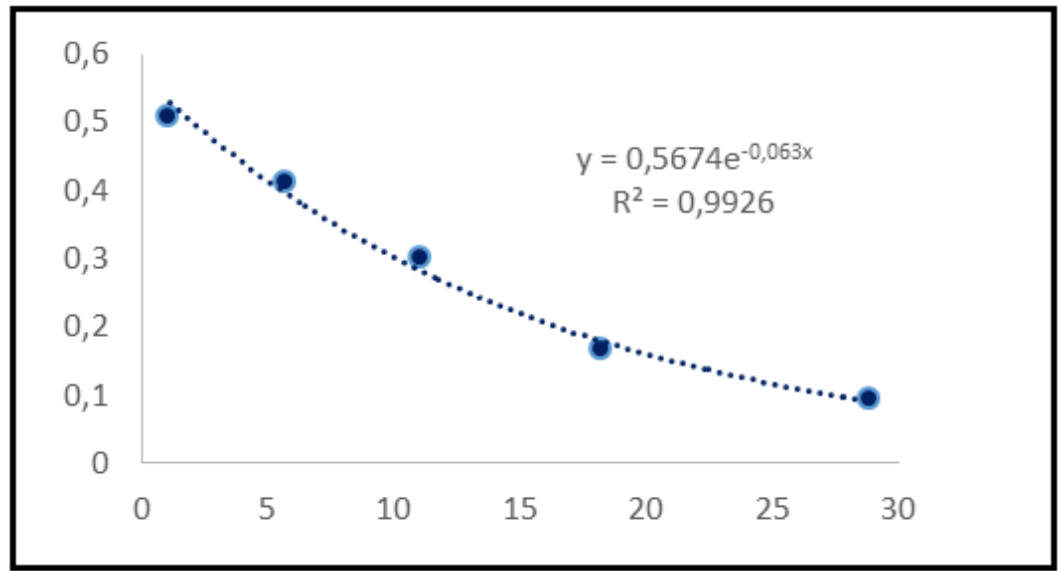

Fuente: autor

La meta es deducir las caracteristicas del movimiento oscilatorio amortiguado:

a. que ya no es periódico; y

b. que la velocidad instantánea decrece de manera exponencial.

El primer análisis, según los datos de la Tabla 1, consiste en calcular el valor de los distintos intervalos de tiempo - para dos valores consecutivos -. En la terceira columna de dicha tabla se aprecia que dichos intervalos no son constantes, sino que aumentan. Así, se vislumbra que el movimento indagado no es periódico; o que el tiempo que tarda el sistema en dar una revolución es cada vez mayor.

El segundo examen se refiere al tipo de decrecimiento de la velocidad tangencial. Mediante el análisis estadístico de correlación de los datos experimentales presentado en la Figura 5, y empleando como modelo de ajuste a una función exponencial, se observa que el coeficiente de correlación - $\mathrm{R}^{2}$ - indica que existe un muy buen grado de ajuste entre los valores experimentales y el modelo matemático.

En consecuencia, y mediante estas dos últimas actividades, los estudiantes podrían llegar a comprender que existe una relación entre el movimento circular 
uniformemente variado y el movimiento oscilatorio amortiguado; particularmente, por la disminución de la velocidad - de manera exponencial -, y por el aumento del tiempo en sus oscilaciones.

\section{CONCLUSIONES}

Este trabajo tiene el objeto de incorporar el smartphone - como sensor - para las experiencias en el laboratorio de Física mediadas por TIC.

Una de las virtudes del uso del teléfono es que constituye, actualmente, una herramienta casi imprescindible para los jóvenes. Así, resulta razonable que éstos puedan aprovecharlo para la gestión de su proceso de enseñanza aprendizaje.

Por otra parte, en esta experiencia se incluye la medición de la Fuerza g, concepto asociado técnicamente a la ingeniería. Como la app cuenta con información al respecto, el alumno puede aceder a información relevante y así gestionar parte de sus conocimientos con cierto carácter autónomo. De todos modos, es indudable que debe estar guiado por el docente para poder tener éxito, dado que éste debe emplear sus saberes para diseñar las secuencias didácticas mediante un dispositivo donde sus alumnos cuentan con mayor experticia en su manejo.

Particularmente, en esta propuesta experimental se sostiene la hipótesis de que los estudiantes podrían mejorar su comprensión sobre el movimiento circular uniformemente variado, mediante los análisis descriptivos - o cualitativos - y cuantitativos - a través de la selección, ajuste y procesamiento de los datos experimentales -. Como consecuencia, también lograrían una asociación entre la experiencia descrita en este trabajo, con las características de un sistema que describe una oscilación amortiguada. Todos estos contenidos suelen ser de gran relevancia en la fomación de los futuros ingenieros.

Para finalizar, esta propuesta educativa puede continuarse mediante varias actividades, dado que el uso de los smartphones invita a desarrollar distintas tareas com el objeto de favorecer el proceso de enseñanza - aprendizaje de la Física. 


\title{
Analysis of non-uniform circular motion by using smartphones as sensors
}

\begin{abstract}
This paper presents a didactic proposal to analyze the circular movement of a platform commonly used in the study of rotations. It is considered that smart cell phones can be used as technological tools by engineering students, favoring significant learning of physics through the use of ICT. The experience uses as sensor a smartphone with an application (app) with free access. Force $g$ is measured in the three dimensions of space $(x ; y ; z)$, after calibration thereof and selection of the Cartesian coordinate system. The data collected are corrected, and then proceed to qualitative and quantitative analysis, in order to explain the behavior of the platform - smartphone system. As a novelty, the relationship between uniformly decelerated circular motion and damped oscillatory motion is incorporated, similar to that between the uniform circular and the simple harmonic.
\end{abstract}

KEYWORDS: Circular movement; Practical Jobs; Smartphone; Physics Teaching. 


\section{Análise de movimento circular não uniforme usando smartphones como sensores}

\section{RESUMEN}

Este artigo apresenta uma proposta didática para analisar o movimento circular de uma plataforma comumente utilizada no estudo de rotações. Considera-se que os telefones inteligentes podem ser utilizados como ferramentas tecnológicas pelos estudantes de engenharia, favorecendo a aprendizagem significativa da física através do uso das TIC. A experiência utiliza como sensor um smartphone com uma aplicação (app) de acesso livre. A força g é medida nas três dimensões do espaço ( $x ; y ; z)$, após sua calibração e seleção do sistema de coordenadas cartesianas. Os dados coletados são corrigidos e, em seguida, proceder a análises qualitativas e quantitativas, a fim de explicar o comportamento do sistema plataforma - smartphone. Como novidade, a relação entre movimento circular uniformemente desacelerado e movimento oscilatório amortecido é incorporada, similar àquela entre a circular uniforme e a harmônica simples.

PALABRAS - CHAVE: Movimento Circular; Trabalhos praticos; Smartphones; Ensino de Física. 


\section{REFERENCIAS}

ALMIRON, M. y PORRO, S. Las TIC en la enseñanza: un análisis de casos. Revista electrónica de investigación educativa, v. 16, n.2, p. 152-160, 2014.

AVELEYRA, E. et al. Desarrollo de material educativo para estudiar un péndulo simple con MYUDUTU. Edutec. Revista Electrónica de Tecnología Educativa, v. 38, 187, p. $1-13,2011$.

CALDERÓN S. et al. Aulas-laboratorios de bajo costo, usando TIC. Revista Eureka sobre Enseñanza y Divulgación de las Ciencias, v. 12, n.1, p. 212-226, 2015.

ENRIQUE, C.M. et al. Dinámica de Sistemas Oscilantes: Concepciones en estudiantes de ingeniería. Enseñanza de la Física (Memorias 14은 SIEF), v. 30, Número especial, p. 63 - 72, 2018.

GARCÍA BARNETO, A. y GIL MARTÍN, R. Entornos constructivistas de aprendizaje basados en simulaciones informáticas. Revista Electrónica de Enseñanza de las Ciencias, v. 5, n. 2, p. 304 - 322, 2006.

GONZÁLEZ, M. A., et al. Uso de smartphones en experimentos de Física en el laboratorio y fuera de él. Departamento de Física la Materia Condensada, Escuela de Ingenierías Industriales, 2015.

KUHN J., y VOGT P. Applications and examples of experiments with mobile phones and smartphones in physics lessons. Frontiers in Sensors, v. 1, n. 4, p. 6773, 2013.

LENDOIRO VALLE, B. Desarrollo y evaluación de una App Android para la medida de dosis de vibraciones en tractores. Trabajo Fin de Grado, Universidad de Valladolid. Escuela Técnica Superior de Ingenieros de Telecomunicación, 2017.

MAGGIO, M. El tutor en la educación a distancia. En: Litwin, E. (compiladora): La educación a distancia. Colección Agenda Educativa. Buenos Aires: Amorrortu Editores S. A., 2000.

MARTíNEZ PÉREZ, J. Obtención del valor de la aceleración de la gravedad en el laboratorio de física.Experiencia comparativa del sensor de un teléfono celular inteligente y el péndulo simple. Revista Eureka sobre Enseñanza y Divulgación de las Ciencias, v. 12, n. 2, p. 341-346, 2015.

Página | 48

VOGT P., y KUHN J. Analyzing radial acceleration with a smartphone acceleration sensor. The Physics Teacher, v. 51, n. 3, p. 182-183, 2013. 
SANS J. et al. Oscillations studied with the smartphone ambient light sensor. European Journal of Physics, v. 34, n. 6, p. 13-49, 2013.

SEARS, F.; ZEMANSKY, M.W.; YOUNG, H.D., y FREEDMAN, R. A. Física Universitaria. Addison - Wesley: México, 2009.

Recebido: 21 de novembro de 2018.

Aprovado: 21 de dezembro de 2018.

DOI:

Como citar: ENRIQUE, C.M. Análisis del movimiento circular no uniforme mediante el uso de smartphones como sensores, Revista Brasileira de Física Tecnológica Aplicada, Ponta Grossa, v. 5, n.2, p. 37-49, dezembro. 2018

Contato: CLAUDIO MARIO ENRIQUE: cenrique@frsf.utn.edu.ar

Direito autoral: Este artigo está licenciado sob os termos da Licença Creative Commons-Atribuição 4.0 Internacional.

(c) (1) 